\section{Chile en el debate sobre la forma de la Arquitectura Moderna}

Hugo Mondragón Profesor, Escuela de Arquitectura, Universidad Diego Portales

A mediados del s. XX, Sudamérica -especialmente Brasil- fue blanco frecuente de las miradas de la prensa arquitectónica, en pleno debate del Movimiento Moderno. En ese contexto, la práctica chilena era vista como un elemento menor; este artículo presenta los proyectos nacionales que participaron en la discusión impulsada por las revistas de la época.

ARquiteCtURA CHILENA EN LAS REVISTAS DE EE.UU. y EuRopa, 1937-1955 / Entre enero y febrero de 1943, con el auspicio de la Oficina de Asuntos Interamericanos (OIAA) ${ }^{1}$, se presentó en el Museo de Arte Moderno de Nueva York la exposición Brazil builds. Es muy posible que este hecho haya incrementado el interés de los editores norteamericanos y europeos por la arquitectura brasileña en particular y por la de América Latina en general; lo cierto es que desde finales de los años treinta, al menos una importante revista norteamericana de arquitectura se había comenzado a interesar por la producción arquitectónica de México.

En once años (1937-1948) siete revistas de Europa y EE.UU. ${ }^{2}$ publicaron catorce números dedicados de manera importante o exclusiva a la arquitectura de países latinoamericanos; esto sin mencionar grupos más pequeños de obras y proyectos desarrollados en la región, que de manera persistente poblaron las páginas de revistas francesas, inglesas y norteamericanas desde fines de los años treinta hasta mediados de la década del cincuenta.

En 1955 la división de arquitectura del MoMA, bajo la dirección de Philip Johnson, publicó el libro de Henry-Russell Hitchcock Latin American architecture since 1945. Con él se cerró un ciclo caracterizado por el interés que las publicaciones especializadas del hemisferio norte mostraron por la producción arquitectónica de la región.

CHILE EN LAS REVISTAS DE ARQUITECTURA NORTEAMERICANAS Y EUROPEAS. 1937-1955 / Si se compara con México, Brasil o Argentina, los proyectos y obras de arquitectura hechos en Chile no fueron particularmente atractivos -o permanecieron desconocidos- para los editores europeos y norteamericanos especializados. De las siete revistas registradas sólo tres (Arts \& Architecture, Tecniques et Architecture y Architectural Review) incluyeron alguna obra de arquitectura que se podía asociar con Chile. De ellas, sólo una (Arts \& Architecture) publicó sobre Chile en más de una ocasión: en agosto de 1943, "Post-war house" un proyecto de Emilio Duhart e I.M. Pei que obtuvo el segundo lugar en un concurso; 5 meses más tarde, se publicó "House for the post-war worker", un nuevo proyecto desarrollado por la dupla Duhart y Pei, y en octubre de 1944, "A comercial fishing village in Chile”. Éste era el proyecto con el que Emilio Duhart, bajo la dirección de Sergio Larrain García-Moreno, se había titulado de arquitecto en la Universidad Católica de Chile. En mayo de 1947 Tecniques et Architecture publicó “Residence au pied des cordillères” de Ignacio Tagle Valdés, una construcción de montaña ejecutada en madera; en agosto de 1948 Architectural Review publicó el Hogar Hipódromo de Chile bajo el título "Amusement centre at Santiago, Chile" atribuido únicamente a Enrique Gebhard. Finalmente, en abril de 1949, Arts \& Architecture publicó "Laborers house development in Chile" del arquitecto Horacio Acevedo. Interesa examinar aquí la manera como cada una de estas obras participa como propuesta en los debates desarrollados al interior de las revistas acerca de las condiciones formales de la Arquitectura Moderna, para mostrar que, contra lo que tradicionalmente se sostiene, tampoco desde
While the debate on Modern Movement developed in the middle of $20^{\text {th }}$ century, South America -especially Brazil- was a regular subject for international press. At that time Chilean practice was often seen as secondary; this article reviews local projects participating in the discussion that architectural magazines motivated.

el punto de vista del estilo es posible referirse a la Arquitectura Moderna como una unidad homogénea.

LAS OBRAS, LOS AUTORES Y LOS DISCURSOS FORMALES / El lenguaje propuesto por Duhart y Pei en sus dos proyectos está dominado por un orden modular que es subsidiario de la voluntad de alcanzar una pieza arquitectónica que pudiera ser prefabricada y producida industrialmente. De esto se deriva una estética de la repetición que informa a la envolvente y a la planta de los proyectos (fig. 01)

Espacialmente los proyectos mostraban una preferencia por el espacio horizontal, continuo y fluido, que se conseguía gracias al enfrentamiento de dos planos horizontales (piso y techo) desprovistos de cualquier tipo de desniveles y sin elementos de soporte vertical como protagonistas de la escena espacial (figs. 02 y 03).

En "Post-war house" los autores propusieron como tipo edificatorio un pabellón de dos pisos de planta estrictamente rectangular, al que se conectaba una cabina de servicios tangencialmente. En "House for the post-war worker” propusieron un pabellón de planta centralizada subdividida en 9 cuadrados iguales. En el cuadrado central dispusieron el módulo de servicios de la casa. En volumen, la propuesta contemplaba un volumen central de mayor altura (módulo de servicios) envuelto circularmente por un volumen de menor altura (estar, comedor y habitaciones). En paralelo, y conectado por un pasillo cubierto, se ubicaba un volumen cúbico de menor tamaño donde estaba el estacionamiento y un pequeño taller (fig. 05).

El orden dimensional primario que cualificaba las relaciones entre interior y exterior estaba determinado por el tamaño de los paneles prefabricados, utilizados como elementos de cierre y división interior. La ausencia de uno o varios paneles construía una transparencia visual total entre interior y exterior; en ambos casos, la losa del piso se apoyaba discreta pero intencionadamente sobre unos dados de hormigón que funcionaban como pilotis recortados, gracias a los que la casa parecía flotar sobre el terreno (figs. 06 y 07).

En el proyecto para un pueblo pesquero en el sur de Chile, Emilio Duhart realizó una propuesta formal en la que se mezclaron principios racionales con aproximaciones pintorescas y una importante cuota de lenguaje regional. La dimensión racional estuvo presente en la estética de la repetición que salpicaba los dibujos de Duhart; no sólo en el plano del plan maestro, donde las viviendas aparecieron dibujadas como cuadrados negros que se repetían a lo largo de la red de caminos propuesta, sino también en los dibujos en perspectiva en que Duhart se encargaba de destacar la estructura modular y repetitiva de los edificios.

La dimensión pintoresca del proyecto aparecía con más fuerza en el trazado de la planta del poblado (fig. 08). Las sinuosas circulaciones obedecían a la voluntad de crear una secuencia de perspectivas múltiples y evitar la calle recta; de alguna manera, el trazado aproximaba la propuesta más a un parque inglés que a un hecho urbano. También había 
01 - 03 Arts \& Architecture, agosto de 1943. "Post-war house" de Duhart y Pei

04 Arts \& Architecture, abril de 1949. "Laborers house development in Chile" de Horacio Acevedo

05 - 07 Arts \& Architecture, enero de 1944. "House for the post-war worker" de Duhart y Pei

08 - 10 Arts \& Architecture, octubre de 1944. "A comercial fishing village in Chile". Pueblo pesquero en canal de Ofqui, proyecto de título de Emilio Duhart

11 - 12 Techniques et Architecture, mayo de 1947. "Residence au pied des cordillères" de Ignacio Tagle Valdés

13 - 15 The Architectural Review, agosto de 1948. "Amusement centre at Santiago, Chile". Hogar Hipódromo de Chile de Enrique Gebhard y Jorge Aguirre
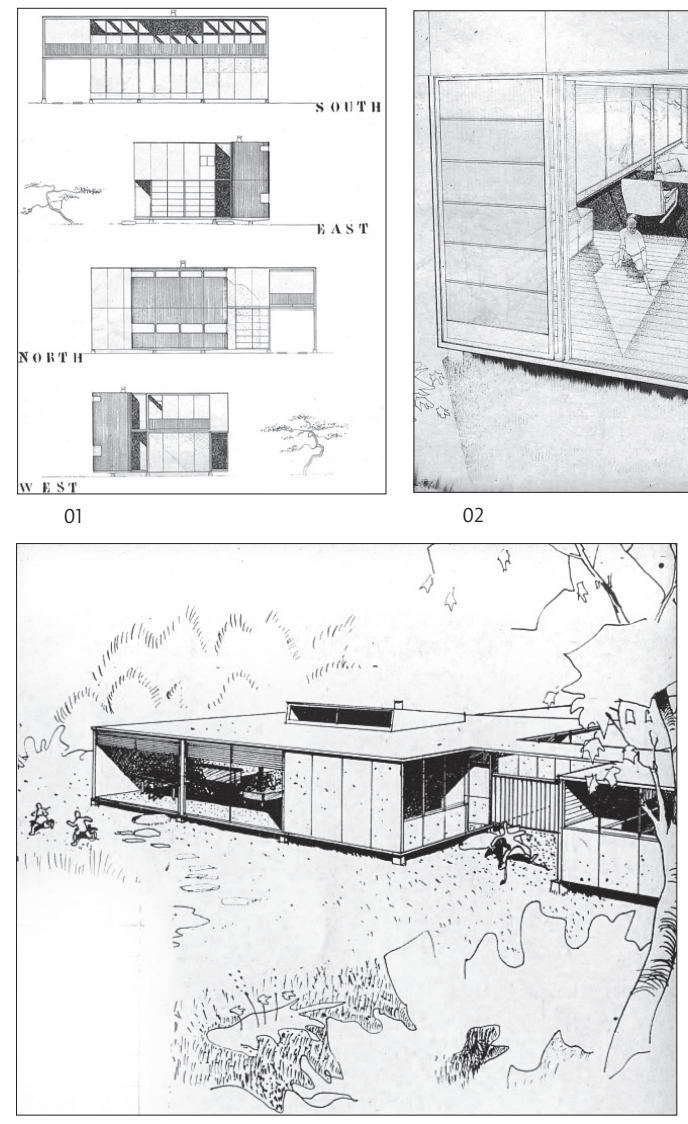

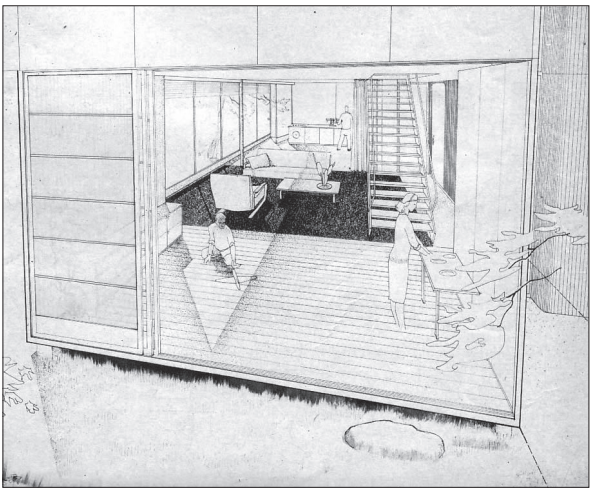

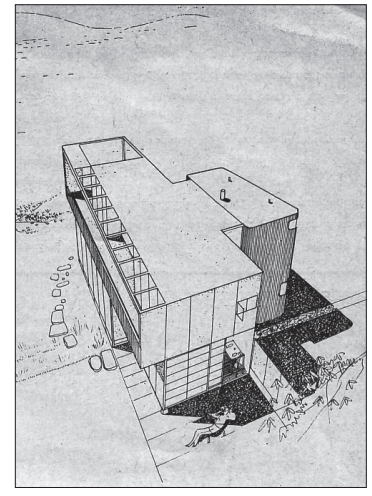

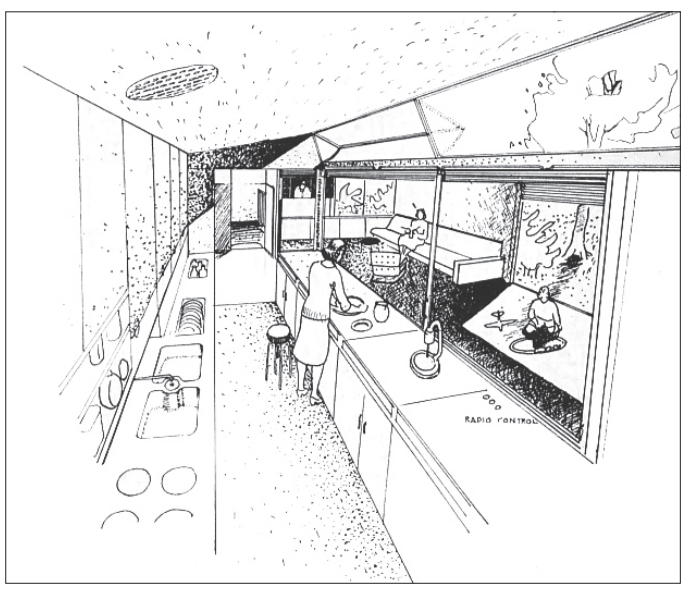

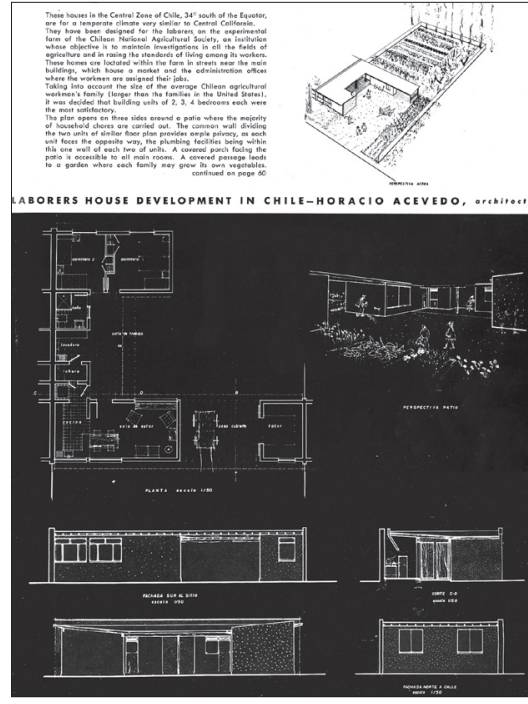

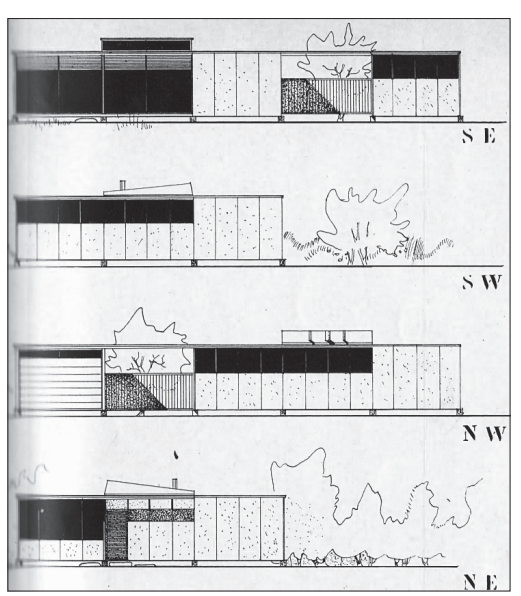

07 un cierto pintoresquismo en la disposición de los edificios que configuraban la plaza central (fig. 09), que no obedecen a un orden geométrico fácilmente discernible: más bien parecen buscar un efecto visual sugerente. Las enormes terrazas con taludes de la plaza principal recuerdan cierta arquitectura precolombina; por otra parte, muchos de los dibujos de la propuesta estaban poblados de guiños náuticos. En el diseño de la torre de la iglesia, Duhart deliberadamente utilizó una cita al mástil de una embarcación, todas operaciones que hablan de la voluntad de mímesis de la arquitectura propuesta con el paisaje náutico existente. El lugar se hizo presente en la determinación material en piedra y madera, a la usanza de la arquitectura de la zona, adoptando elementos figurativos de la arquitectura local: techos muy inclinados, aleros, pórticos, pasarelas cubiertas, torres de chimeneas y ventanas (fig. 10). Sometido a estas tensiones, Duhart logró desarrollar un lenguaje potente, articulado, contemporáneo y singular en su propuesta, sin acudir automáticamente al canon establecido para la Arquitectura Moderna, que conocía bastante bien.

La casa para trabajadores proyectada por Horacio Acevedo proponía un lenguaje figurativo expresado de modo elemental: los muros, desprovistos de decoración, escondían cubiertas levemente inclinadas dando a los volúmenes la apariencia exterior de prismas; tres tipos de ventanas correspondían a los tres niveles de privacidad de la casa (áreas social, privada y de servicios). Espacialmente, la casa proponía una interesante solución de espacios abiertos pero cubiertos que graduaban y cualificaban el tránsito entre el interior y el exterior, erosionando además el perímetro (fig. 04).

La casa en la cordillera de Ignacio Tagle Valdés, cerca de Santiago, no se expresó en el lenguaje abstracto más o menos codificado por el que se conocía a la Arquitectura Moderna de la época. Contrariamente, empleó un lenguaje figurativo de cubiertas a dos aguas, balcones, balaustradas, aleros, desniveles y ventanas que sin embargo no coincide con los códigos impostados de los estilos históricos: se trata de un lenguaje arcaico poderosamente primitivo. La casa tiene el mismo gesto rotundo y genuino de las máscaras africanas que tanto fascinaron a los artistas de vanguardia en Europa de entreguerras (fig. 11). Es cierto que la sala de estar presentaba un espacio con doble altura que podía ser atractivo, pero sin duda lo más llamativo del proyecto era cierta componente expresiva que comunicaba de manera simple y directa las preguntas esenciales que el proyecto intentaba responder (fig. 12). El proyecto para el Hipódromo de Enrique Gebhard era el que más se acercaba a lo que para la época se había canonizado como el lenguaje de la Arquitectura Moderna. Dominaban los volúmenes prismáticos simples. El acceso estaba dispuesto hacia un costado de la planta, lo que obligaba a tener una aproximación asimétrica al edificio y un encuentro igualmente asimétrico con el espacio interior. Al ingresar, el espacio interior se tensionaba diagonalmente por el cuerpo escultórico de la escalera helicoidal en primer plano a la izquierda y la doble altura del restaurante al final a la derecha. El espacio interior estaba punteado por unos pilares estructurales dispuestos regularmente pero, como en las villas del primer Le Corbusier, también aquí el espacio y la estructura estaban separados lingüísticamente. La planta estaba compuesta por tres cuerpos independientes: un cuerpo central de mayor tamaño y figura rectangular, y dos cuerpos más pequeños que se disponían en la periferia de manera asimétrica tensionando centrífugamente el cuerpo principal. Los techos dominantes eran planos y se utilizaban como terrazas: el contrapunto estaba a cargo de la bóveda en cáscara de hormigón. El lenguaje de fachadas se articulaba a partir de sistemas de orden establecidos por grillas de distintos tamaños (figs. 13 a 15).

Conclusión / Es evidente que desde el punto de vista formal, las obras publica- 


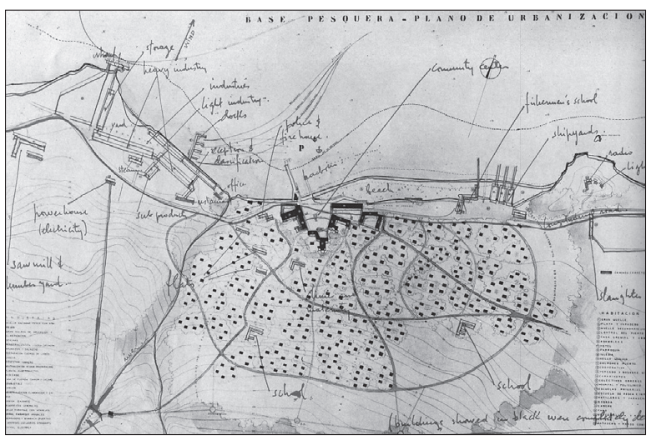

08

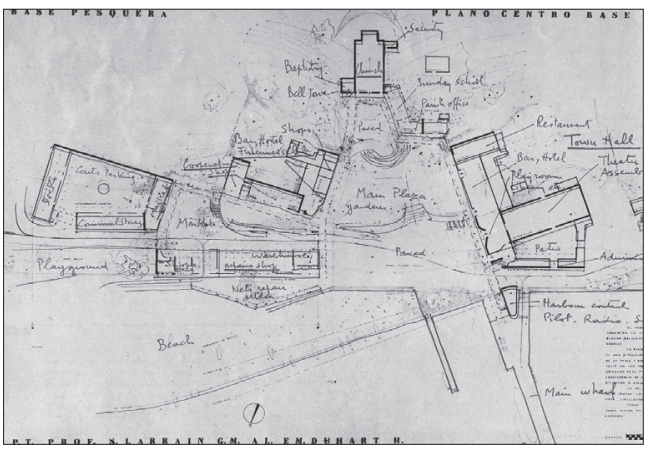

09

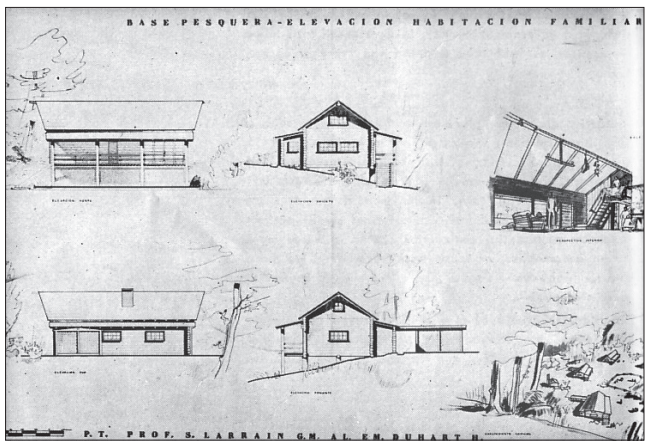

10

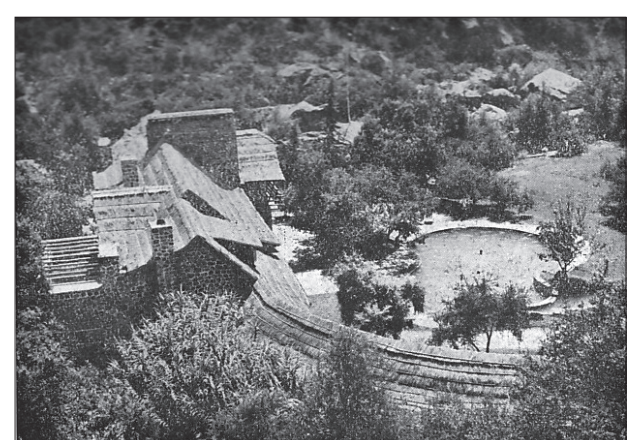

11

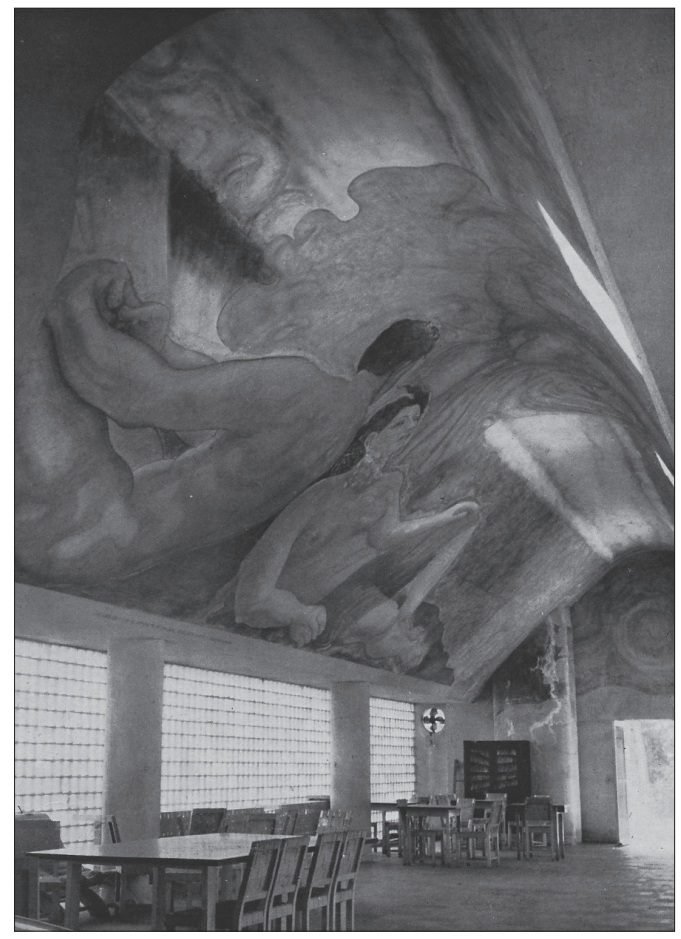

13

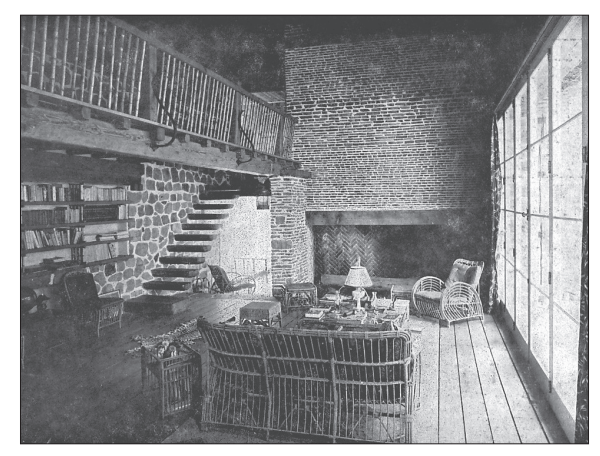

12
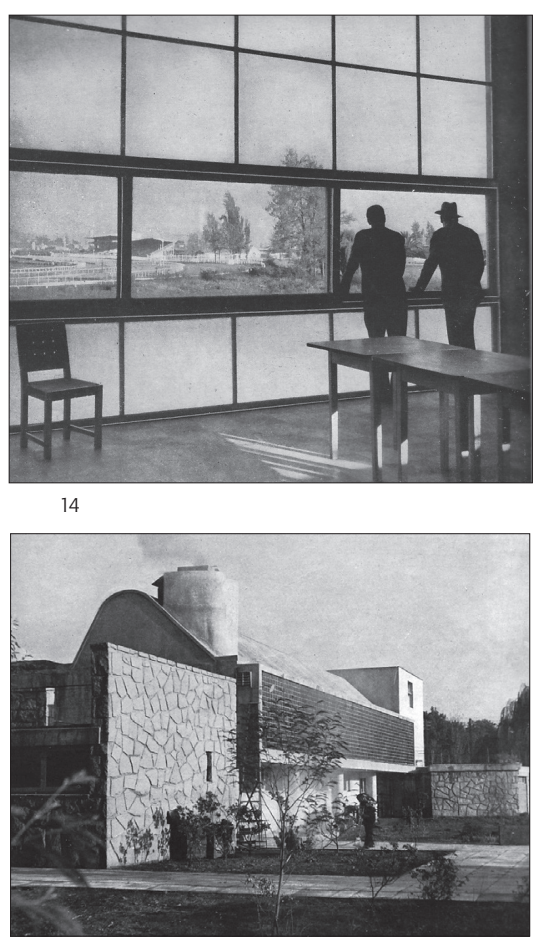

das difícilmente logran construir una unidad estilística homogénea. Contrariamente, es posible identificar al menos cuatro tipos de planteamiento en relación al lenguaje formal de la Arquitectura Moderna: modernismo canónico, modernismo primitivo y arcaico, modernismo esencial y modernismo mestizo. El valor de la muestra reside precisamente en su diversidad. Construye una imagen para ilustrar la tesis propuesta por la historiadora Sarah Goldhagen según la cual la Arquitectura Moderna ha de ser vista como un discurso abierto y sin fin, al interior del cual se han venido escenificando desde hace unos 100 años una serie de debates motivados por los intentos de responder preguntas ontológicas, en los que participan arquitectos, críticos e historiadores

Las obras de arquitectura chilena publicadas en las revistas de EE.UU. y Europa pueden ser vistas como respuestas creativas y sugerentes, que participaron en un debate internacional provocado por la siguiente pregunta: ¿Cómo debe lucir un edificio moderno? ARo

Agradecimientos Del aUtor

Al doctorado en Arquitectura y Estudios Urbanos de la P.U.C., por haber apoyado financieramente mi viaje de estudios al G.S.D. en Harvard University entre diciembre de 2005 y marzo de 2006 en el contexto del desarrollo de mi tesis doctoral A la Loeb Library del G.S.D. en Harvard University por haberme permitido consultar sus archivos de revistas. De allí fueron tomadas la totalidad de las imágenes que

\section{Bibliografía}

Goldhagen, Sarah. "Something to talk about". Journal of the Society of Architectural Historians, JSAH Vol. 64 № 2. Society of Architectural Historians, Chicago, junio de 2005.

\section{Listado de artículos y revistas}

"New Architecture in México”. The Architectural Record Vol. 81 № 4. F. W. Dodge
Corporation, Nueva York, abril de 1937. | "Architecture of Brazil”. The Architectural Record Vol. 93 № 1. F. W. Dodge Corporation, Nueva York, enero de 1943. / "Brazil builds for the new world”. Arts \& Architecture Vol. 60 № 2. John D. Entenza, Los Ángeles, febrero de 1943. / "Post-war house”. Arts \& Architecture Vol. 60 № 8. John D. Entenza, Los Ángeles, agosto de 1943. / "House for the post-war worker". Arts \& Architecture Vol. 61 № 1. John D. Entenza, Los Ángeles, enero de 1944. / "Brazil”. The Architectural Review № 567. The Architectural Press, Surrey, marzo de 1944. | "A comercial fishing village in Chile". Arts \& Architecture Vol. 61 № 10. John D. Entenza, Los Ángeles, octubre de 1944. / "Jorge Kalnay”. The Architectural Record Vol. 98 № 4. F. W. Dodge Corporation, Nueva York, octubre de 1945. / "Recent Brazilian work”. Progressive Architecture Vol. 27 № 4. Reinhold Publishing Corp., Nueva York, abril de 1946. / "Brazil builds a new city: cidade dos motores". Progressive Architecture Vol. 27 № 9. Reinhold Publishing Corp., Nueva York, septiembre de 1946. / "South America I”. Architectural Forum Vol. 85 № 5. Time Inc., Nueva York, noviembre de 1946. Selección de arquitectura de Colombia y Venezuela. / "South America II”. Architectural Forum Vol. 86 № 2. Time Inc., Nueva York, febrero de 1947. Selección de arquitectura de Argentina. / "Residence au pied des cordillères". Techniques et Architecture № 5-6. Tecniques et Architecture, París, mayo de 1947. I “Modern Mexico". The Architectural Record Vol. 102 № 1. F. W. Dodge Corporation, Nueva York, julio de 1947. | "Brèsil”. L'architecture d'aujourd'hui № 14. L'architecture d'aujourd'hui, París, septiembre de 1947. | "Brazil”. Architectural Forum Vol. 87 № 5. Time Inc., Nueva York, noviembre de 1947. / "Argentine”, "Brèsil”. L'architecture d'aujourd'hui № 16. L'architecture d'aujourd'hui, París, enero de 1948. / "South America III”. Architectural Forum Vol. 88 № 6. Time Inc., Nueva York, junio de 1948. Selección de arquitectura de Uruguay. | "Amusement centre at Santiago, Chile". The Architectural Review № 620. The Architectural Press, Surrey, agosto de 1948. / “Laborers house development in Chile”. Arts \& Architecture Vol. 66 № 4. John D. Entenza, Los Ángeles, abril de 1949. 